Ann. Biol. anim. Bioch. Biophys., I966, 6 (3), 315-332.

\title{
MÉCANISME DE LA PÉNÉTRATION ET VOIE D'ACHEMINEMENT DES GRAISSES DANS LA CELLULE ABSORBANTE DE L'INTESTIN GRÊLE DU PORC
}

\author{
X. VODOVAR, J. FLANZY \\ avec la collaboration technique de Françoise PEssey \\ Station centrale de Nutrition, \\ Centre national de Recherches zootechniques, 78 -Jouy-en-Josas
}

SOMMAIRE,

La pénétration des graisses ingérées à travers la membrane plasmique libre de la cellule absorbante du Porc et leur acheminement à travers le cytoplasme ont été observés en microscopies photonique et électronique. L'administration des graisses a été faite soit par ingestion normale, soit par infusion dans un segment isolé de la première partie de l'intestin grêle. Pour parvenir dans les espaces intermicrovilleux, les graisses sont préalablement fractionnées en particules dont le diamètre n'excède pas $\mathrm{o}, \mathrm{I} \mu$. La présence des graisses, pendant toute la durée de l'absorption, au fond des espaces intermicrovilleux, dans la membrane au même niveau et dans la zone apicale, ainsi que leur absence à l'intérieur des microvillosités indiquent leur voie principale de pénétration. La substance osmiophile qui imprègne la membrane est formée de très petites particules dont le diamètre n'excéderait pas $5^{\circ} \AA$, ce qui suggère que la pénétration à travers la membrane se ferait sous forme de molécules ou tout au plus d'association de quelques molécules. Dès leur entrée dans la zone apicale, les graisses se rassemblent à l'intérieur du RE par lequel elles sont acheminées jusqu'à leur évacuation dans les espaces intercellulaires ou à la base de la cellule.

\section{INTRODUCTION}

Les molécules des graisses alimentaires, constituées presque exclusivement par les esters des acides gras et du glycérol, sont apolaires, hydrophobes, insolubles dans l'eau. Les produits qui apparaissent au cours de la digestion des triglycérides et l'état physico-chimique sous lequel ils pénètrent dans la cellule épithéliale absorbante ont fait l'objet de nombreuses recherches. BERNARD (I856) fut le premier à démontrer l'action du suc pancréatique sur l'émulsification de l'hydrolyse des graisses neutres avant leur absorption. Selon HOPPE-SEYLER (I877), 1'hydrolyse intestinale ne serait que partielle et 1'absorption se ferait sous forme d'émulsion (ceci concorderait avec les observations histologiques, car les graisses ingérées se trouvent dans la cellule épithé- 
liale sous forme de gouttelettes). PFLÜGER (IgOo), au contraire, fut le défenseur de la théorie de 1'hydrolyse complète des triglycérides alimentaires et de leur absorption. exclusivement sous forme d'acides gras et de glycérol. Enfin, les résultats obtenus par FRAZER (I944), selon lesquels l'hydrolyse intestinale des triglycérides resterait incomplète, sont, à présent, définitivement confirmés (Bromstrand, BorGSTröm, DAHLBACH, I959; DESNUELLE et SAVARY, I963). L'équilibre entre les différents produits de l'hydrolyse, acides gras libres, monoglycérides, diglycérides et triglycérides, a été étudié et exprimé sous forme de pourcentage par de nombreux chercheurs (Desnuelife et Constantin, I952; Mattson et al., I952; Borgström, I952 ; FAvarger, r959). Ces produits de la digestion, tout comme les triglycérides, sont insolubles ou peu solubles dans l'eau (ceci malgré leur groupement polaire, car le caractère hydrophobe de ces substances, appelées amphiphiles, est plus fort que leur attraction hydrophile) et se trouvent sous forme de particules dans le contenu intestinal. Selon la théorie de FRAzER (I944), ces particules, dails leur dernière phase intestinale, sont une association d'acides gras libres et de glycérides avec des sels biliaires, et c'est sous cette forme que ces particules, dont le diamètre est inférieur à $0,5 \mu$, pénètrent à travers la membrane plasmique. Pour Hofmann et BorGSTröm (I962, I 964), les acides gras libres et les monoglycérides provenant de 1'hydrolyse s'associeraient avec les sels biliaires sous forme de micelles mixtes. Ces particules, dont le diamètre serait compris entre 30 et roo $\AA$, sont dispersées dans la phase aqueuse du contenu intestinal et c'est à partir de cette pseudo-solution que l'absorption a lieu (Johnston et Borgström, I964).

Dans une publication récente de CLÉMENT (I964), les lecteurs trouveront une revule complète de ce qui a été fait, à ce jour, sur la digestion et l'absorption des graisses du point de vue biochimique. D'autres mises au point, sur ce sujet, ont été faites par Favarger (r959), Feli (ig63), Wotton (I963), Gounelle (I964), Senior (Ig64), BRowN (I964), SWYNGHEDAUW (I965).

A la lumière des différents travaux, on peut constater que les méthodes biochimiques et biophysiques actuelles, malgré leurs perfectionnements constants, ne permettent pas de suivre, d'une manière précise et continue, l'acheminement des graisses à travers la membrane et le cytoplasme de la cellule absorbante. L'hypothèse selon laquelle l'état physico-chimique des graisses, au moment de leur pénétration à travers la membrane plasmique, est le même que dans le contenu intestinal, est basée sur des présomptions. La localisation du siège des transformations physicochimiques à l'intérieur de la cellule absorbante et l'isolement des produits formés se révèlent difficiles ou inopérants. C'est surtout par le prélèvement de la lymphe intestinale, au cours de la digestion, dans le canal thoracique ou dans un tronçon du mésentère, et par l'identification de l'état chimique (grâce aux éléments marqués) des produits absorbés que la plupart des hypothèses sur les transformations intervenues dans la cellule épithéliale absorbante ont été formulées.

L'étude histologique et histochimique de la cellule épithéliale de l'intestin, pendant 1'absorption des graisses, a permis à GRUBY et DELAFOND (I844) de constater que les graisses se retrouvent sous forme de gouttelettes à l'intérieur du cytoplasme de la cellule absorbante. Depuis, ce fait a toujours été confirmé. Les essais effectués notamment par BAKER (I942, 195I) pour déterminer histologiquement le mécanisme et la forme physico-chimique de pénétration des graisses à travers la membrane, n'ont 
pu donner de résultats concluants. La faible résolution en microscopie photonique en est une des causes.

I a microscopie électronique. grâce à son haut pouvoir de résolution et malgré son application relativement récente, a déjà fourni d'importantes précisions sur la morphologie de la cellule absorbante. La membrane plasmique, les microvillosités, la zone apicale et le réticulum endoplasmique ont été plus particulièrement étudiés (GRANGER et BAKer, I949, I950 ; ZitTterQVist, I956 ; PALAY et KARLin, I959 a. ; Vodovar et Flífion, sous presse). La structure complexe de ces éléments aide à comprendre les difficultés que présente l'étude de l'absorption des graisses. Les observations, en microscopie électronique, sur l'acheminement des graisses à travers la membrane et le cytoplasme de la cellule épithéliale ne sont pas toujours concordantes. Pour certains morphologistes (WEISS, I955 ; PALAY et KARLIN, I959 b. ; AsHWORTH, Stembridge et Sanders, Ig60 ; Ladman, Padykula et Strauss, I963; Ashworth et Johnston, I963; PALAY et REVEL, I964), la pénétration des graisses se fait, en grande partie sinon en totalité, en gouttelettes par " pinocytose " à partir des espaces intermicrovilleux. L'argument en faveur de cette hypothèse est la présence des graisses absorbées dans l'endoplasme et quelquefois, dans la zone apicale, sous forme de gouttelettes entourées d'une membrane que 1'on suppose souvent provenir d'une invagination de la membrane plasmique. Ie fait que la graisse, au moment del'absorption, n'ait pu être mise en éviđence à l'intérieur des microvillosités est, aussi, favorable à cette hypothèse. Pour d'autres (LACY et TAYLor, Ig62; SjöSTrARD, I963; ROSTGAARD et BARRNETT, I964, I965), la pénétration se fait sur toute la surface de la membrane plasmique, sous forme de particules très fines qui diffusent à l'intérieur de la microvillosité ét von't se rassembler dans l'endoplasme. La majetıre partie de ces travaux a été effectuée sur le Rat, quelques-uns sur l'Homme.

En résumé, on peut dire que, d'une part, les recherches dans le domaine de la digestion et de l'absorption des graisses alimentaires ont fait, en particulier au cours des quinze dernières années, des progrès importants mais que, d'autre part, de nombreux problèmes sont restés, en partie ou en totalité, sans solution.

- Les produits de la digestion des graisses alimentaires sont-ils absorbés par toute la surface libre de la cellule épithéliaie, ou bien les espaces intermicrovilleux soint-ils des endroits privilégiés, étant donné la structure particulière de la zone apicale en dessous de ces espaces?

- Quels sont les mécanismes et la forme de pénétration de ces produits à travers la membrane plasmique?

- La pinocytose est-elle le mécanisme spécifique dans l'absorption des graisess ou bien un processus occasionnel de peu d'importance sur la quantité des graisses absorbées?

- Par quel processus s'effectue le transport des graisses à travers la zone apicale, puis l'endoplasme de la cellule épithéliale absorbante et quel est le rôle joué dans ce transport par le réticulum endoplasmique?

Les questions énumérées, faisant partie des problèmes non résolus à l'heure actuelle, feront l'objet des présentes observations. Le Porc, notre animal d'expérience habituel, sur lequel, par ailleurs, aucune recherche semblable n'a été faite jusqu'à ce jour, a été choisi pour ces observations. 


\section{MATÉRIEI, ET MÉTHODES}

\section{Matériel}

l.es fragments de l'intestin grêle (du premier tiers du jéjunum-iléon) ont été prélevés sur des porcs de race Large White, d'âge variable, auxquels ont été administrées des graisses par différents procédés.

Les produits utilisés sont des graisses d'origine animale et végétale, des triglycérides homogènes et des acides gras libres (tabl. r).

\section{TABLEAU I}

Liste des graisses pour lesquelles des observalions morphologiques ont été faites au cours de lcur absorption

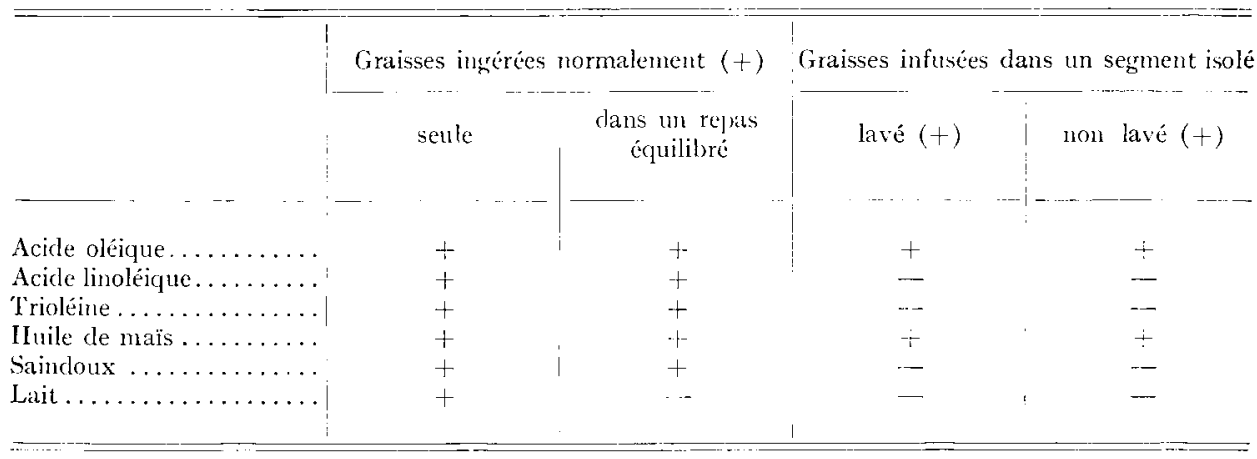

\section{Methodes}

L'administration des graisses expérimentées a été pratiquée suivant différents procédés :

$I^{\circ}$ Les animaux en croissance (après sevrage), mis au régime lipidoprive pendant 48 heures, puis privés de nourriture, excepté d'eau, pendant I 2 heures, ont été nourris, soit 30 minutes, soit I heure avant le sacrifice avec:

a) un repas équilibré en 'glucides et protéines, augmenté d'une graisse expérimentale (tabl. I) en quantité variable, comprise entre 5o et ro g par repas (pour la microscopie photonique) ;

b) une de ces graisses, seule, à raison de $\mathbf{I} g$ environ par $\mathrm{kg}$ de poids corporel (pour microscopies photonique et élcctronique). tions.

Les animaux témoins ont reçu, dans les deux cas, un repas sans graisses, dans les mêmes condi-

$2^{\circ}$ Les porcelets, au cours de l'allaitement, ont été sacrifiés I 5 minutes et 30 minutes après ingestion, soit de lait maternel (pris par tétée ou au biberon), soit d'huile de maīs, à raison de $2 \mathrm{~g}$ par $\mathrm{kg}$ de poids corporel.

$3^{\circ}$ Les porcelets, d'âge différent, après un jeûne allant de 6 à 18 heures, ont été anesthésiés, puis ouverts, soit ventralement, soit latéralement (côté droit); un segment d'environ $25 \mathrm{~cm}$ a été isolé dans le premier tiers du jéjunum-iléon, sans lavage ou après lavage (pour éliminer les substances pouvant s'y trouver et, en particulier, les sels biliaires et le suc pancréaticue) ; il y a été infusé, avec de la solution de Ringer, $5 \mathrm{ml}$ d'huile de maïs ou d'acide oléique, préalablement émulsionnés (par passage dans un homogénéiseur) ou non, additionnés ou non de taurocholate de sodium. Ėnviron I 5 minutes après l'infusion, le segment isolé a été prélevé.

Des graisses de différentes natures et des procédés d'administration variables ont été pratiqués pour essayer de préciser l'influence que certains éléments (sels biliaires, suc pancréatique, taurocholate de sodium, emulsifiants, etc.) peuvent avoir au cours de la pénétration et de l'acheminement de.s graisses. 
Les prélèvements destinés à la microscopie photonique ont été faits suivant le procédé décrit précédemment (VODOVAR, I964). Il a été, en outre, à titre de comparaison, procédé au lavage des pièces prélevées, soit avec du sérum physiologique, soit avec de l'alcool à $50^{\circ}$ ou $70^{\circ}$, pour éliminer les substances intraluminaires et, en particulier, pour essayer d'éliminer les graisses se trouvant dans les espaces intermicrovilleux. Les tissus ont été coupés au cryostat International Company, d'une part, sans fixation préalable, et d'autre part, après un séjour des tissus dans le formol-calcium de Baker, pendant 6 à 12 heures. La coloration des lipides a été faite au noir Soudan B en solution alcoolique à $60^{\circ}$ (Lison, 1950; CaSSELMAN, 1962), celle des tissus au carmin aluné. Jua coloration, en vue de distinguer les acides gras (en particulier l'acide oléique) et les glycérides, a été faite au Bleu de Nil (CAIN, I950) sur les coupes attenantes à celles colorées au noir Soudan.

Pour les observations en microscopie électronique, les tissus ont été prélevés sur les mêmes fragments que ceux utilisés pour la microscopie photonique. La fixation a été effectuéc.

a) au tétroxyde d'osmium à I p. Ioo, tampon véronal-acétate, et à 2 p. I00, tampon phosphate de Milloning, pendant I à 2 heures.

b) au glutaraldéhyde à $6 \mathrm{p}$. 100 , avec post-fixation dans le tétroxyde d'osmium à $2 \mathrm{p}$. Ioo.

La déshydratation a été faite soit avec le méthanol, soit avec l'éthanol, soit avec l'acétone (ce dernier nous a donné le meilleur résultat en ce qui concerne la préservation des lipides).

Les fragments de tissus d'environ $1 \mathrm{~mm}^{3}$ ont été inclus dans le méthacrylate prépolymérisé ou dans l'Epon.

Les coupes ont été obtenues au microtome Porter-Blum, à une épaisseur de 500 à I $000 \AA$.

Les tissus ont été contrastés à l'acétate d'uranyle pendant 30 minutes ou I heure, ou avec l'acétate d'uranyle pendant 30 minutes, puis le citrate de plomb pendant io minutes (Reynolos, $196.3)$.

\section{OBSERVATIONS}

\section{A - Microscopie photonique}

Io Chez les animaux sacrifiés 30 minutes après l'ingestion de l'une des graisses expérimentées (tabl. I), seule ou incorporée à un repas équilibré, des fractions de bol alimentaire sont parvenues dans le premier tiers du jéjunum-iléon. Sur les coupes de tissu, les particules soudanophiles sont présentes simultanément sur le sommet des microvillosités (mv), dans le fond des espaces intermicrovilleux et à l'intérieur du cytoplasme supranucléaire de la cellule absorbante (fig. 2, 3). Quantitativement, le nombre des particules est surtout élevé dans les cellules épithéliales bordant les parties distales des villosités; cependant, et surtout chez les jeunes porcelets, dont les villosités sont encore espacées (VoDOVAR, I964), on trouve des particules soudanophiles sur les coupes des cellules bordant la partie médiane des villosités; elles sont rares ou absentes dans les cellules qui tapissent la surface basale de la villosité.

a) Sur le sommet des mv, les particules sont de taille variable, échelonnée entre plusieurs microns et la limite de la visibilité en microscopie photonique.

b) Les particules soudanophiles occupant le fond des espaces intermicrovilleux sont placées, sauf exception, au même niveau, donnant ainsis, sur la coupe, l'aspect d'une rangée (fig. 2,3 ). Cet aspect est semblable pour les graisses de différente nature.

La nature histochimique de la substance soudanophile engagée dans les espaces intermicrovilleux a été étudiée pour toutes les graisses expérimentées. Dans le cas des acides gras, ingérés séparément, et tout particulièrement pour l'acide oléique (coloration spécifique avec le Bleu de Nil), on peut affirmer que cette substance est constituée, à ce niveau, exclusivement d'acides gras libres. Pour les triglycérides, on peut simplement dire que cette substance représente un mélange de glycérides et 
d'acides gras libres, les méthodes histochimiques actuelies ne permettant pas une évaluation quantitative valable, et ceci d'autant moins que ce rapport nous a paru souvent variable pour une même graisse, au même niveau du jéjunum-iléon.

La présence constante et abondante de substance soudanophile au fond des espaces intermicrovilleux, durant la phase de pénétration des graisses dans la cellule absorbante (même dans le cas où les tissus ont été lavés, au moment du prélèvement, dans du sérum physiologique, à $37^{\circ} \mathrm{C}$, ou avec de l'alcool à $50^{\circ}$ oul $70^{\circ}$ ) pourrait être interprétée de façon contradictoire. On peut supposer, soit que le fond des espaces intermicrovilleux est le lieu privilégié ou exclusif de la pénétration des graisses à travers la membrane plasmique de la cellule absorbante, soit que ces espaces sont la partie de la surface libre de la membrane plasmique où l'absorption est réduite ou inexistante, ce qui justifierait l'accumulation des graisses.

Pour essayer d'élucider ce problème, nous avons, sous anesthésie, infusé au porcelet, dans un segment isolé du premier tiers du jéjunum-iléon, $3 \mathrm{ml}$ d'huile de maïs émulsionnée. Dix minutes après l'infusion, le segment est rapidement lavé pour éliminer l'huile de maïs intraluminaire non absorbée; des fragments de muqueuse sont alors prélevés; puis environ trois minutes plus tard, le segment est prélevé en entier. I,es tissus des deux prélèvements sont coupés, colorés et observés. Dans la partie prélevée immédiatement après lavage, les particules soudanophiles sont abondantes dans le fond des espaces intermicrovilleux; en revanche, sur les coupes des tissus prélevés trois minutes environ après lavage du segment, les particules soudanophiles sont rares ou complètement absentes. Après plusieurs expériences semblables, nous avons toujours obtenu le même résultat. Ceci nous permet de conclure que les fonds des espaces intermicrovilleux sont les parties de la surface libre de la cellule où l'intensité de l'absorption est très grande. Pour des raisons qui seront exposées ultérieurement, nous pensons que les graisses pénètrent par cette surface d'une façon préférentielle sinon exclusive.

c) A l'intérieur de la cellule absorbante, la substance soudanophile est surtout abondante dans l'endoplasme supranucléaire, où elle se trouve sous la forme de gouttelettes plus ou moins grandes et d'aspect variable suivant la nature de la graisse ingérée. La zone apicale est plus claire et on n'y distingue pas de substance soudanophile, en microscopie photonique.

$2^{\circ}$ Une heure après l'ingestion des graisses, la substance soudanophile est abondante dans les espaces intercellulaires, dans le stroma et dans la lactéale, tandis que persiste l'image précédente.

$3^{\circ}$ L'aspect et la répartition de la substance soudanophile dans les tissus prélevés sur des animaux ayant absorbé l'une des graisses soit seule, soit incorporée à un repas équilibré, ne présentent pas de différences.

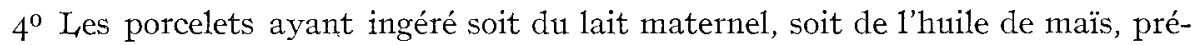
sentent une image semblable aux précédentes.

Chez les animaux témoins de chaque groupe, qui n'ont pas ingéré de graisses, il n'a jamais été trouvé de substance soudanophile dans la partie supranucléaire de la cellule absorbante.

$5^{\circ}$ Les coupes de tissus prélevés sur des animaux auxquels $1^{\prime}$ huile de maïs ou 1'acide oléique ont été administrés sous anesthésie et suivant des conditions variables, 
dans un segment isolé du premier tiers đu jéjunum-iléon, ont donné lieu aux observations suivantes:

a) dans le cas où les graisses, sans émulsification préalable, ont été infusées avec de la solution de Ringer dans le segment isolé, sans lavage préalable de ce dernier, l'absorption présente l'aspect précédemment décrit, mais son intensité (quantitéappréciée de substance soudanophile dans les espaces intermicrovilleux et le cytoplasme) est plus faible.

b) si les graisses sont infusées comme précédemment, sans émulsification préalable, mais après lavage au sérum physiologique ( 9 p.I ooo de $\mathrm{ClNa}$ à $\left.39^{\circ} \mathrm{C}\right)$ du segment isolé, 1'absorption est très réduite et, sur de nombreuses coupes, nulle.

c) si les graisses, après émulsification mécanique légère (les particules présentent une gamme de diamètres compris entre $3 \mu$ et la limite de visibilité en microscopie photonique), sont infusées dans un segment non lavé, l'absorption est comparable à celle trouvée sur les animaux ayant ingéré les graisses normalement, trente minutes avant le sacrifice.

d) pour les graisses préparées comme précédemment et infusées dans le segment préalablement lavé, l'absorption est semblable ou parfois légèrement moins intense que dans le cas précédent.

e) pour les graisses auxquelles il a été incorporé du taurocholate de sodium avant leur émulsification et leur incorporation dans un segment non lavé, l'absorption est très intense.

f) les conditions précédentes étant semblables, mais le segment isolé étant lavé préalablement, 1'absorption des graisses est normale.

Pour l'ensemble de ces observations, il n'a pas été vu de différences dans l'intensité de l'absorption entre 1'huile de maîs et l'acide oléique. Quant aux résultats, et en particulier ceux du groupe $d$, nous reviendrons ultérieurement sur leur signification.

\section{$\mathrm{B}$ - Microscopie électronique}

Rappelons que les tissus ont été prélevés sur les mêmes fragments d'intestin grêle que pour l'observation en microscopie photonique et que les prélèvements provenant d'animatıx ayant ingéré, au dernier repas, des graisses incorporées à des glucides et à des protéines, n'ont pas fait l'objet de ces observations pour permettre une localisation plus précise des graisses.

\section{Io Graisses en contact avec les mv avant leur pénétration.}

D'une façon générale, sur les coupes observées en microscpie électronique, la présence de la substance osmiophile sur la bordure extérieure des mv et dans les espaces intermicrovilleux est peu abondante par rapport à ce qui a été observé en microscopie photonique, sur des coupes appartenant aux mêmes fragments de tissu. La différence de préservation des lipides, dans les deux cas, est due aux techniques de préparation des tissus et au confectionnement des coupes. En effet, en ce qui concerne la microscopie photonique, la préservation des lipides dans les tissus congeiés et coupés au cryostat est presque totale. En ce qui concerne la microscopie électronique, certaines méthodes récentes permettent une conservation satisfaisante des lipides 
intercellulaires (IDELMAN, I964) grâce, en partie, au support protéique de ceux-ci ; mais les lipides extracellulaires, qui n'ont pas ce support, se détachent facilement et sont éliminés partiellement ou en totalité.

Lorsqu'on trouve des particules osmiophiles au sommet des mv, elles ont une forme et une taille variable. Latéralement, entre deux mv contiguës, la substance osmiophile, sous forme de pellicule, accolée à la membrane des $\mathrm{mv}$, est souvent abondante (fig. 4, 5, 6). Entre les parties basales des $\mathrm{mv}$ et au fond des espaces intermicrovilleux, on trouve, d'une façon irrégulière, une ou plusieurs particules osmiophiles, de forme généralement sphérique, au diamètre compris entre 500 et I $000 \AA$, rarement supérieur. Dans ces espaces, on trouve souvent les emplacements d'où ont été éliminés les lipides au cours du confectionnement des coupes (fig. 5, 6).

\section{$2^{\circ}$ Aspect de la membrane plasmique bordant les mv.}

a) Au sommet des mv, la membrane plasmique au moment de l'absorption, présente un aspect presque toujours effiloché et on y trouve, à ce niveau, des éléments osmiophiles disposés de façon telle qu'ils donnent l'impression d'être engagés dans la membrane plasmique (fig. $4,5,6$ ). On pottrait présumer qu'il s'agit de pénétration des lipides sous forme de particules dont le diamètre serait de l'ordre de 30 à $50 \AA$. Cependant, le fait que ces particules ne se retrouvent pas à l'intérieur de la mv, ni entre les couches osmiophiles de la membrane plasmique, amène à penser qu'il s'agit de particules de graisse à l'intérieur des mucosités qui enrobent l'extrêmité des mv. A ce niveau, les attaches des fibres centrales des mv avec la couche intérieure osmiophile de la membrane plasmique (VODOVAR et Fiśchon, sous presse) rendent cet aspect encore plus complexe.

b) Lans la membrane plasmique bordant latéralement les $\mathrm{mv}$, on distingue les 3 couches classiques sans substance entre les deux lamelles osmiophiles (fig. 7). Les couches interne et intermédiaire sont sans différence apparente avec celles des tissus prélevés sur les animaux témoins, tandis que la couche externe, contre laquelle la substance osmiophile est souvent accolée, donne une image plus épaisse et plus dense. Cependant, à aucun moment, à ce niveau, on n'a observé d'infiltrations de substance osmiophile à l'intérieur de la membrane.

c) La membrane plasmique qui borde les parties basales des mv et le fond des espaces intermicrovilleux est souvent imprégnée de particules osmiophiles, dontla taille, quoique difficile à préciser, n'excéderait pas $50 \AA$. Cette image, observée notamment autour des gouttelettes de graisses préservées au fond des espaces intermicrovilleux et aux endroits de leur emplacement, représente, sans aucun doute, la phase de franchissement de la membrane par les graisses (fig. 4, 5).

Le fond des espaces intermicrovilleux, au moment de l'absorption, présente un alignement moins régulier que chez les animaux à jeun ; cependant ces invaginations, plus ou moins fréquentes, restent, sans exception, d'une faible profondeur de l'ordre de $O, I \mu$.

\section{$2^{\circ}$ Acheminement des graisses à travers le cytoplasme de la cellule absorbante.}

a) A l'intérieur des mv, sur aucune de nos coupes et quelle que soit la technique de préparation des tissus, nous n'avons pu mettre en évidence de substance osmiophile, dont on puisse dire qu'elle provient des graisses absorbées. 
b) Dans la zone apicale, en commençant immédiatement sous la membrane plasmique du fond des espaces intermicrovilleux, la substance osmiophile, en quantité importante, est répartie d'une manière irrégulière et variable suivant les coupes (fig. 4, 5, 6). Cette substance, dans la plupart des cas, est sans forme précise et s'étale suivant un axe en direction de l'endoplasme. Plus rarement on observe des gouttelettes dont le diamètre varie entre 250 et $650 \AA$, isolées ou en succession. Pour l'ensemble l'image montre un acheminement continu de la substance osmiophile, de densité variable, de la membrane plasmique à l'endoplasme.

Une cytomembrane, plus ou moins apparente, moins épaisse et moins dense que la membrane plasmique, limite soit tout autour, soit latéralement la substance osmiophile (fig. 4, 5,6). Cette présence de graisses à l'intérieur des tubules ou des vésicules suivant l'incidence des coupes, indique que les graisses, dès leur franchissement de la membrane plasmique, se trouvent à l'intérieur du RE, ce qui a déjà été observé chez

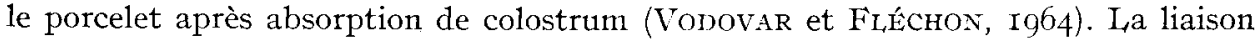
entre le RE et la membrane plasmique au fond des espaces intermicrovilleux n'a pu être mise en évidence d'une manière satisfaisante. Par contre, la continuité du RE de la zone apicale et de l'endoplasme s'observe couramment (fig. 5, 6, 7).

c) La substance osmiophile, (fig. 4, 5, 6) change d'aspect dès son passage dans l'endoplasme. Entre la zone apicale et la région de Golgi, sous forme plus ou moins sphérique, les gouttelettes de graisse sont, suivant 1'incidence des coupes, entourées complètement ou partiellement par le RER. Le diamètre interne de ce dernier, qui représente la voie d'acheminement des graisses, est élargi de plusieurs fois aux endroits où la graisse est présente. La taille des gouttelettes est variable sur une même coupe avec un diamètre généralement compris entre I ooo et 2 ooo $\AA$ (fig. 5, 8, 9). Leur nombre varie d'une cellule à l'autre et pour une même cellule suivant les coupes.

Pout les tissus fixés au tétroxyde d'osmium et colorés à l'acétate d'uranyle, le contraste de la substance osmiophile est le même dans la zone apicale que dans dans l'endoplasme (fig. 5, 8, 9). Dans le cas de coupes colorées au citrate de plomb, la substance osmiophile est plus claire dans l'endoplasme que dans la zone apicale.

d) Dans la zone de Golgi, les gouttelettes de graisse, tout en restant d'aspect semblable, sont moins nombreuses que dans la zone sub-apicale.

Les vésicules golgiennes en relation avec le RER se remplissent de graisse pendant l'absorption (fig. 9) ; leur volume devient nettement supérieur à celui des vésicules des animaux témoins n'ayant pas absorbé de graisse.

A partir des vésicules golgiennes. les gouttelettes de graisse continuent à être acheminées par le RER, la plupart en direction latérale, et sont déposées dans les espaces intercellulaires, à des niveaux variables, ce qui est rendu possible par la liaison entre le RER et la membrane plasmique latérale (fig. Io). D'autres gouttelettes, bien moins nombreuses, sont acheminées à côté du noyau (fig. Io), dans la partie subnucléaire, puis vers le stroma.

Une faible partie des graisses se retrouve dans les espaces intercellulaires, immédiatement à partir des desmosomes situés sous la bandelette obturante, échappant ainsi au transit golgien. A partir de la région golgienne, le diamètre des gouttelettes diminue irrégulièrement (fig. IO) ; parvenues dans les espaces intercellulaires, ces gouttelettes ne sont plus incluses dans le $\mathrm{RER}$ et ont un diamètre variable, compris généralement entre 500 et I ooo $\AA$ (fig. Io, II). 
L'accumulation des graisses dans les espaces intercellulaires est d'autant plus importante qu'on s'approche de la base de la cellule (fig. II). Ceci permet de supposer que le passage à travers la basale est relativement lent.

Remarquons la présence, en général peu fréquente, de la substance osmiophile à 1'intérieur de l'endoplasme, sous forme étalée (fig. 5, 8). Cette forme, déjà signalée par PALAY et REVEL, (I964), nous semble être la conséquence d'un déchirement de la membrane réticulaire au cours de la préparation des tissus, qui se produit surtout au niveau des anastomoses de RER. I1 n'est cependant pas exclu qu'il s'agisse de graisses dont la voie de pénétration n'est pas la même que celle des graisses se trouvant dans la lumière du RER.

Signalons enfin que les graisses administrées par des procédés différents présentent la même image morphologique.

\section{DISCUSSION}

Il nous semble souhaitable de revenir sur certaines des observations décrites précédemment, soit pour les discuter, soit pour les préciser.

a) Dernière phase intraluminaire des graisses ingérés :

La substance soudanophile, présente d'une façon permanente au cours de l'absorption dans le fond des espacess intermicrovilleux est, de toute évidence, la dernière phase intraluminaire de la graisse ingérée. Cette phase, dont il est rarement question, est souvent peu apparente en microscopie électronique, à cause de l'élimination des lipides au cours de la préparation des tissus. Pour éviter les interprétations erronnées à ce sujet, il est nécessaire de pratiquer sur le même fragment de tissu les observations en microscopie électronique et en microscopie photonique. Les graisses parvenues dans les espaces intermicrovilleux, tout en étant encore tne phase intraluminaire, représentent, nous semble-t-il, une étape irréversible et particulière dans le processus de l'absorption des graisses. Flle n'est pas récupérable dans le contenu intestinal par le lavage habituel de la muqueuse et, de ce fait, échappe au biochimiste en tant que substance intraluminaire. Cela montre en particulier combien il est aléatoire de discuter de la forme physico-chimique des graisses au moment de leur passage à travers la membrane plasmique libre en tenant compte, seulement, de leur état dans le contenu intestinal.

Pour que les graisses puissent parvenir entre les microvillosités, il est nécessaire qu'elles soient fractionnées en particules dont le diamètre n'excède pas celui des espaces intermicrovilleux, qui est d'environ o, I $\mu$. Il est important de souligner que le procédé de fractionnement ne semble pas avoir d'influence sur les différentes étapes de l'acheminement des graisses. Ainsi, celles que nous avons administrées après émulsification mécanique en absence de sels biliaires sont retrouvées dans les espaces intermicrovilleux et à l'intérieur de la cellule, de façon comparable à celles dont la digestion se fait en présence de sels biliaires.

b) Lieu de pénétration des graisses ingérées à travers la membrane plasmique libre (microvillosités, espaces intermicrovilleux) de la cellule absorbante :

La structure de la membrane plasmique est considérée, à l'heure actuelle, comme identique sur toute la surface libre de la cellule absorbante (SJösTrAND, Ig63; VoDo- 
VAR et FLÉCHON, sous presse) et on pourrait s'attendre à ce que la pénétration des graisses, dans des conditions normales, s'effectue de la même façon sur toute la surface libre de la cellule. Or, au cours de nos observations, nous avons constaté que la membrane plasmique couvrant les espaces intermicrovilleux était seule à être imprégnée, d'une façon évidente, par des particules osmiophiles provenant des graisses ingérées.

La structure attenante à la membrane plasmique libre est différente selon que l'on considère le sommet de la microvillosité, sa partie latérale ou le fond des espaces intermicrovilleux. Rappelons que nous n'avons pas trouvé de substance osmiophile ni dans les fibres des microvillosités, ni dans le cytoplasme situé entre ces fibres et la membrane. Ces observations concordent avec 1'ensemble des travaux effectués sur ce sujet jusqu'à ce jour (PALAY et KARLIN, I959 b; PALAY et REVEL, I964; AsHWORTH et Johnston, I963; Pheips, Rubin et LUFT, I964, etc...) à l'exception toutefois de la publication de LACY et TAYLoR (I962) qui signalent, chez le Rat, la présence de particules osmiophiles à l'intérieur des microvillosités et entre les deux couches opaques de la membrane plasmique. Ces observations semblent s'attacher à des cas isolés et demanderaient à être confirmées. Par contre, les tissus attenants à la membrane couvrant la surface intermicrovilleuse sont abondamment pouvus de substance osmiophile, ce qui nous incite à nous demander si la différence de structure des tissus attenants à la membrane plasmique libre n'aurait pas une influence sur le lieu de pénétration des graisses.

Quoi qu'il en soit, cette pénétration dans la cellule absorbante se fait, d'une façon évidente, à travers la membrane qui couvre les espaces intermicrovilleux. Pourtant, une hypothèse, selon laquelle une part moins importante des graisses pourrait pénétrer par la membrane de la microvillosité sous forme de molécules qui ne se rassembleraient qu'au moment de leur pénétration dans le $\mathrm{RE}$, reste possible.

c) État physico-chimique du produit de la digestion des graisses au moment de leur franchissement de la membrane plasmique libre :

Les controverses au sujet de la forme physique sous laquelle le produit de la digestion des graisses neutres pénètre à travers la membrane plasmique libre ne sont pas, à l'heure actuelle, terminées. L'opinion de la majorité des chercheurs est partagée entre la théorie de FRAZER (I944, I946, I954, I958), selon laquelle les graisses sont absorbées à l'état d'émulsion et la conception des auteurs suédois (HoFmans et Borgström I962 ; Johnston et Borgström, I964; Borgström I963), d'après laquelle la pénétration à travers la membrane se ferait à l'état de micelles formées au cours de la digestion et exclusivement en présence de sels biliaires. Les propriétés des particules micellaires, notamment leur pseudo-solubilité dans l'eauet leur taille inférieure à celles des particules des émulsions, sont, suivant BoRGSTRÖM (I962) des arguments en faveur de cette forme d'absorption.

Au cours d'une précédente étude (Vodovar et FLÉchon, sous presse) et des présentes observations, nous avons pu constater que les espaces intermicrovilleux, chez le Porc, avaient un diamètre de 0 , I $\mu$ environ ; cela signifierait que, dans les cas normaux, des particules de taille supérieure, notamment celles de l'émulsion définie par FRAZER $(0,5 \mu)$ ne pourraient accéder aux espaces intermicrovilleux, ni être absorbées par les cellules.

L'acheminement des particules osmiophiles par invagination de la membrane plasmique, dont l'extrémité se détache dans le cytoplasme sous forme de vésicules 
(BENNE'TT, I956) est pratiquement inexistant chez le Porc, ce qui permet, à notre avis, de considérer coinme négligeable la quantité de graisses absorbées par cette voie. Constatons, cependant, que nos observations sont en désaccord avec celles de PALAY et KARLIN, (I959 b), ; PALAy et ReVEL (I964), ; Ashworth et Johnston (I963) etc., pour lesquels, chez les espèces étudiées par eux, la pinocytose est considérée comme la voie principale de la pénétration des graisses dans la cellule absorbante.

La substance osmiophile, mise en évidence au cours de l'absorption, dans la membrane plasmique couvrant les espaces intermicrovilleux ne serait pas en contradiction avec la pénétration des graisses sous forme de micelles, ou tout au moins sous forme de particules de quelques molécules associées soit avant, soit au moment de la pénétration, et dont le diamètre n'excéderait pas $50 \AA$. En revanche, nos constatations, à savoir que les graisses émulsionnées et infusées dans un segment isolé de l'intestin pénètrent dans les cellules absorbantes d'une façon normale en l'absence de sels biilaires, éliminés par lavage, ne concordent pas avec la théorie micellaire. En effet, si la formation de micelles se fait exclusivement en présence de sels biliaires (HofMAnn et Borgström, rg62), les graisses, dans le cas de nos expériences, pénètrent bien dans la cellule absorbante sous une forme qui n'est pas micellaire. Nos observations corroborent les résultats obtenus par d'autres chercheurs (PESSOA et $a l .$, I953 ; Cohen, Ig6r ; Hauboi ${ }_{4}$ et $a l$., I962 ; Razumov, I963 ; MorGan, I964, etc.) concernant les graisses de différente nature et expérimentées chez des espèces différentes. Le fait que les graisses émulsionnées sont normalement absorbées en l'absence de sels biliaires suggère que le rôle principal de ceux-ci, dans les phénomènes de digestion et d'absorption des graisses, est l'émulsification (FrRAZER, I944; ARVy, I958). Un autre point de la théorie suédoise qui demanderait à être précisé est celui de savoir à quel niveau se désagrège la micelle mixte (sels biliaires, monoglyeérides, acides gras) pour libérer les sels biliaires qui seront absorbés vers la fin du jéjunumiléon (BAKER et SEARI,E, Ig60) et ce que deviennent les monoglycérides et les acides gras libres. Restent-ils à l'état moléculaire ou forment-ils une émulsion? On peut supposer, comme le font certains biochimistes (ISSELBACHER et SENIOR, I964) que les micelles sont désagrégées au contact de la muqueuse, mais alors l'absorption se ferait sous forme moléculaire et non micellaire. En conséquence, on peut admettre que l'état micellaire facilite le transport intraluminaire des graisses, mais on ne voit. pas son rôle dans leur passage à travers la membrane. Remarquons aussi que les graisses mises en évidence dans les espaces intermicrovilleux et, en particulier, dans le fond de ces espaces et qui représentent la dernière phase avant 1'absorption (pour une fraction ou pour la totalité des graisses) ne se trouvent pas à l'état micellaire. Ces observations conduisent à penser que les graisses franchissent la membrane plasmique libre à l'état de molécules ou tout au plus d'association de quelques molécules à partir des particules déjà en contact avec la muqueuse. Il semble peu important que ces particules soient sous forme de micelles ou d'émulsion, pourvu qu'elles parviennent dans les espaces intermicrovilleux.

Il reste l'interprétation de la pénétration des graisses émulsionnées en l'absence de suc pancréatique dans le cas du segment isolé et lavé préalablement, ou bien dans le cas d'une dérivation prolongée de ce suc. Dans le premier cas, on peut supposer que les graisses émulsionnées, parvenues dans les espaces intermicrovilleux, sont hydrolysées d'une part par le suc pancréatique échappé au lavage, ou d'autre part par les enzymes membranaires au contact de la membrane plasmique (HAMPToN, I962; 
Razumov, I963). Nous pensons que la seconde supposition pourrait s'appliquer à tous les cas où le suc pancréatique est absent. D'ailleurs, cela semblerait correspondre au processus qui intervient au cours de la pénétration des graisses dans la cellule adipeuse, ainsi qu'au cours de leur mobilisation (WASSERMANN et Mac IONArd, I963).

d) Acheminement des graisses à travers le cytoplasme de la cellule absorbante :

I. Zone apicale : d'après les présentes observations, la substance osmiophile est mise en évidence, pour la première fois, en quantité importante, dans la zone apicale au cours de l'absorption des graisses. Bien que la visualisation soit souvent rendue difficile et incomplète par la densité élevée et la structure complexe de cette zone, il n'est pas douteux que les graisses, dès leur franchissement de la membrane, soient situées à l'intérieur du RE, par lequel elles sont acheminées vers l'endoplasme et non par "pinocytose "(PALAY et KARLIN, I959 b, ; AshwORTh et Johnston, I963, etc.) ou par diffusion, sous forme de molécules ou de micelles (SJöSTRAND, I963). On peut présumer que la relation entre le RE et le fond des espaces intermicrovilleux se fait soit directement par des pores de faible diamètre, soit indirectement par liaison avec la couche opaque interne de la membrane plasmique ; cependant, tant que cette liaison ne sera pas établie d'une façon plus éviđente, on ne sera pas en mesure de préciser le mécanisme exact par lequel les graisses parviennent dans le RE.

I a forme et la densité des graisses, d'une part, le contraste obtenu par le citrate de plomb, d'autre part, sont différents suivant que les graisses se trouvent dans la zone apicale ou dans l'endoplasme. Il semble que cela corresponde à une différence de leur nature chimique. La zone apicale est, à notre avis, le lieu de resynthèse de produits de la digestion, qui apparaissent dans l'endoplasme sous forme de triglycérides, comme cela a pu être constaté par réaction histochimique.

2. Endoplasme : en ce qui concerne la forme des graisses et leur trajet d'acheminement dans l'endoplasme, nos observations ne concordent qu'en partie avec celles de PaLAY et KarLin (I959 b). En effet, nous avons pu montrer, sans contestation possible, que les graisses, à 1'intérieur du cytoplasme, étaient entourées de RE et non de vésicules provenant de la membrane plasmique.

\section{CONCLUSION}

Io Pendant l'absorption des graisses, le fond des espaces intermicrovilleux est constamment occupé par la substance osmiophile, qui n'est pas éliminée par le lavage habituel de la muqueuse. Pour parvenir à ce niveau, les graisses ont dî être fractionnées en particules n'excédant pas $O, I \mu$ de diamètre. Ce fractionnement, au cours de la digestion, est fait par les sels biliaires, mais leur action peut être remplacée par une émulsification mécanique, sans que des perturbations se manifestent dans le processus de l'absorption.

$2^{\circ}$ Le renouvellement rapide de la substance soudanophile au fond des espaces intermicrovilleux, sa présence dans la membrane plasmique à ce niveau, ainsi que dans la zone apicale attenante, indique que les surfaces intermicrovilleuses sont la voie principale de pénétration des graisses dans le cytoplasme.

$3^{\circ}$ Le franchissement de la membrane plasmique libre se fait sous forme de particules n'excédant pas $50 \AA$ de diamètre et ceci à partir des graisses parvenues au contact 
de cette membrane au fond des espaces intermicrovilleux. La forme micellaire ne semble pas nécessaire à une pénétration normale. La pénétration par " pinocytose " est un phénomène se produisant exceptionnellement chez le Porc.

$4^{\circ}$ L'acheminement des graisses à travers la zone apicale et l'endoplasme en passant par les vésicules golgiennes jusqu'aux espaces intercellulaires se fait à l'intérieur du RE. La jonction entre le RER et la membrane plasmique latérale permet l'évacuation des graisses dans les espaces intercellulaires.

$5^{\circ}$ La liaison entre la membrane plasmique libre et le RE de la zone apicale n'étant pas encore démontrée d'une façon évidente, il est difficile de spécifier la manière dont les graisses parviennent dans le RE.

Reçu pour publication en fésrier 1966.

\section{SUMMARY}

FAT ABSORTION AND TRANSPORT IN THE EPITHELIA CELL

OF SMALL INTESTINE OF THE PIG

The penetration of lipids through the plasmic membrane of the absorbing cell of the Pig and its transport through cytoplasm were studied with classical and electron microscopy. Lipids were either normally ingested or infused in an isolated portion of the intestine. In both cases, lipids were found at the tops of microvilli, between them and in the cytoplasm simultaneously. There are lipids in the folds of microvilli as long as the absorption takes place. To reach this luminal position, lipids must at first be divided into particles the diameter of which should not exceed $0,1 \mu$. Without any bile salt, lipids are normally absorbed if they have been mechanically emulsionized before ingestion. When using electron microscopy, the lipids in the villifolds often are eliminated because of the preparation technique. But, they are never eliminated by the usual washing of the mucous. During absorption, the plasmic membrane looks fringy at the tops of microvilli, and it is impregnated with osmiophile substance between them. We observed that it is in the villifolds where the absorption intensity is the greater. An important amount of lipids was found in the apical zone of the absorbing cell, but none inside the microvilli ; the invaginations of the plasmic membrane are scarce and rather small : the absorption by "pinocytosis " is exceptional in the Pig. In cytoplasm, lipids are always to be found within the endoplasmic reticulum through which they are conveyed to intercellular lumina, some of them at the bottom of the absorbing cell, most of them in Golgi apparatus.

\section{RÉFÉRENCES BIBLIOGRAPHIQUES}

Arvy L., 1958. Les techniques actuelles d'histoenzymologie, les estérases carboxyliques. Biol. méd., 47, I 2-I 60 .

Ashworth C. T., Stembridge V. A., Sanders E., I960. Lipid absorption, transport and hepatic assimilation studied with electron microscopy. Amer. J. Physiol., 198, 1 $326-13^{28}$.

Ashwortin C. T., Johnston S. M., 1963. The intestinal absorption of fatty acid : a biochemical and electron microscopic study. J. Lipid Res., 4, 454-460.

BAKER J. R., I942. The free border of the intestinal epithelial cell of vertebrates. Quart. J. micr. Sci. 84, 73-103.

Baker J. R., I95I. The absorption of lipoid by the intestinal epithelium of the mouse. Quart. J. micr. Sci., 92, 79-86.

Baker R. D., Searle G. W., i960. Bile salt absorption at various levels of rat small intestine. Proc. Soc. exper. Biol., N. Y., 105, $5^{2 \mathrm{I}-5^{2} 3}$

BennetT H. S., 1956. The concepts of membrane flow and membrane vesiculation as mechanisms for active transport and ion pumping. J. biophys. biochem. Cytol., 2, Suppl., 99-103.

BERNARD C., I956 Mémoire sur le pancréas et sur le rôle du suc pancréatique dans les phénomènes digestits, particulièrement dans la digestion des matières grasses neutres. Baillière, Paris.

Blomstrand R., Borgströn 13., Dahlbach O., 1959. Extent of total hydrolysis of dietary glycerides during digestion and absorption in the human. Proc. Soc. exper. Biol., N.Y., 102, 204-206.

Borgström B., I952. On the action of pancreatic lipase on triglycerides in vivo and in vitro. Acta physiol. scand., 25, 328-347. 
BORGSTRÖM B., I962. Digestion and absorption of fat. Gastroenterology, 43, 216-219.

BORGSTRÖm B., I963. Absorption of triglycerides. In : Lipid Transport. Proc. internat. Symp., October 10-11, 1963, 15-31. Ch. Thomas, Springfield.

BRown J. L., I964. The role of monoglycerides in the biosynthesis of triglycerides in the intestine. Thesis Univ. Texas, Dallas, $108 \mathrm{p}$.

CAIN A. J., r950. The histochemistry of lipoids in animal, Biol. Rev., 25, 73-II2.

Casselman W. G. B., 1962. Histochimie. Dunod, Paris, 220 p.

Clément G., I964. La digestion et l'absorption des graisses. J. Physiol., 56, i I I-I92.

Confen B. J., I96r. Fat excretion in dogs lacking both bile and pancreatic juice. Proc. Soc.exper. Biol., N. Y., 107, 40-42.

Desnuelle P., Constanitin M. J., I952. Formation des glycérides partiels pendant la lipolyse des triglycérides dans l'intestin. Biochim. biophys. Acta, 9, 531-537.

Desblelle P., Savary P., ig63. Specificities of lipases. J. Lipid Res., 4, 369-384.

Favarger 1'., I959. L'absorption intestinale et l'utilisation des graisses. In: Wiss. Veröftl. Dtsch. Ges. Ernährung, 59-88, Dietrich Steinkopff, Darmstadt.

FELL B. F., 1963. Epithelial form and function in the intestine. In : Progress in nutrition and allied Sciences, I4I-1 58. Oliver and Boyd,Edimburgh, London.

Frazer A. C., 1944. Differenciation in the absorption of olive oil and oleic acid in the rat. J. Physiol., London, 103, 306-31 2 .

Frazer A. C., I946. The absorption of triglycerides fat from the intestine. Physiol. Rev., 26, 103-119.

Frazer A. C., i 954 . Transport of lipid through cell membranes. In : Symp. Soc.exper. Biol., 8, Activetransport secretion, 490-50 , Union Press, Cambridge.

T'Razer A. C., 1958. Fat absorption and its disorders. Brit. med. Bull., 14, 21 2-219.

Gounelle J. C., I964. L'absorption intestinale des triglycérides. Prod. Prob. Pharm., 19, 367-37I.

Granger B., Baker R. F., 1949. Electron microscope investigation of the striated border of intestinal epithelium. Anat. Rec., 103, 459 .

Granger I3., Baker R. F., i95o. Electron microscope investigation of the striated border of intestinal epithelium. Anal. Rec., 10\%, $423^{-440 .}$

GRUBY, DeLAFond, I 843 . Résultats des recherches faites sur l'anatomie et les fonctions des villositiés intestinales, l'absorption, la préparation et la composition organique du chyle chez les animaux. C. R.Acad. Sci., Paris, 16, I I $94^{-1} 200$.

HAмтton J. C., I962. Observations on the relations between intestinal epithelial cells and cellular components of luminal contents in the distal ileum in the mouse. In: 5ih Internal. Congr. Eleclron Micr., 2, LL-g. Academic Press, Jew York.

Haubold II., Hecer E., Loew W., Ronuschinskyj R., 1962. Histologische Beiträge zur Resorption von Fetten und Fettemulsionen. Frelle, Seifen, Anstrichmittel, 64, 606-6 + 4.

IIOFMANN A. F., Borgström B., rg62. Physico-chemical state of lipids in intestinal content during their digestion and absorption. Fed. Proc., 21, 41-50.

IIOFMANy A. F., Borgström B., I964. The intestinal phase of fat digestion in man. The lipid content of the micellar and oil phases of intestinal content obtained during fat digestion and absorption. J. clin. Invest., 43, $247^{-257}$.

Hoppe-Seyler F., 1877. Physiologische Chemie, Berlin, 347 p.

Idelman S., I964. Modification de la technique de Luft en vue de la conservation des lipides en microscopie électronique. J. Micr., 3, 7r 5-718.

Isselbacher K. J., Sennior J. R., I964. The intestinal absorption of carbohydrate and fat. Gastroenterology, 46, 287-298.

Johnston J. M., Borgström B., ig64. The intestinal absorption and metabolism of micellar solutions of lipids. Biochim. biophys. Acta, 84, 4 I $2-423$.

LACY D., TAYLOR A. B., I I662. Fat absorption by epithelial cells of the small intestine of the rat. Amer. J. Anat., 110, I $55^{-1} 85$.

Ladman A. J., Padykula H. A., Stratss E., 1963. A morphological study of fat transport in the normal human jejunum. Amer. J. Anat., 112, $389-394 .(+\mathrm{I} 2$ planches).

IIson L., I960. Histochimie et cytochimie animales. Tome II, 749-756. Gauthier Villars, Paris.

Mattson F. H., Benedict J."H., Martin J. B., Beck L. W., 1952. Intermediates formed during the ingestion of triglycerides. $J$. Nutr., 48, 335-344.

Morgan R. G. H., I964. The effect of bile salts in the lymphatic absorption by the unanaesthetized rat of intraduodenally infused lipids. Quart. J. exper. Physiol., 49, 457-465.

PaLAY S. L., KarLin L. J., I959 a. An electron microscopic study of the intestinal villi. I. The fasting animal. J. biophys, biochem. Cylol, 5, 363-372.

Palay S. L., Karlin L. J., I959 $b$. An electron microscopic study of the intestinal villi. II. The pathway of fat absorption. J. biophys. biochem. Cytol., 5, 373-384.

Palay S. L., Revel J. P., r 964 . The morphology of the fat transport. In : Lipid Transport. Proc.inlernal. Symp. Oclober 10-11, $1963,33^{-69}$. Ch. Thomas, Springfield.

Annales de Biologie animale. - 1966. 
Pessoa V.C., Krm K.S., Ivy A. C., 1953. Fat absorption in absence of bile and pancreatic juice. Amer. J. Physiol., 174, 209-218.

Pilelps P. C., Rubin C. E., Luft J. H., I 964. Electron microscope techniques for studying absorption of fat in man with some observations on pinocytosis. Gastroenterology, 46, г $34-$ - 55 .

PFLüger E,. I 900. Der gegenwärtige Zustand der Lehre von der Verdauung und Resorption der Fette und eine Verurteilung der hiermit verknüpften physiologischen Vivisectionen am Menschen. Arch. Ges. Phy'siol., $82,3 \circ 3-380$.

Razumov M. I., 1963. Intestinal villi cells and fat assimilation. Fed. Proc., 22, Part II, T 3 I I-T $3^{\mathbf{I}} 3$.

REYNOLDS E.S., 1963 . The use of lead citrate at high $\mathrm{pH}$ as an electron opaque stain in electron microscopy. J. Cell. Biol., 17, 208-212.

RostgaARd J., BarRnetT R. J., 1964. Absorption of lipid as micelles by microvilli of rat small intestine. Anal. Rec., 148, 398 .

ROSTGAaRd J., BARRNETT R. J., 1965. Fine structural observations of the absorption of lipid particles in the small intestine of the rat. Anat. Rec., 152, 325-349.

Senior J. R., I964. Intestinal absorption of fats. J. Lipids Res., 5, 495-521.

Swyngiredauw B., 1965. Métabolisme et techniques d'exploration des triglycérides circulants. Palhol. Biol., 13, 74-99.

Vonovar N., 1964. Intestin grêle du Porc. IT. Structure histologique des parois et plus particulièrement de la tunique muqueuse en fonction de l'agge de l'animal. Ann. Biol. anim. Bioch. Biophys., 4, $113^{-1} 39$.

Vodovar N., FLéchoN J., i 964 . Le réticulum endoplasmique des cellules épithéliales. Une voie de l'absorption intestinale. Ann. Biol. anim. Bioch. Biophys., 4, 199-208.

Vodovar N., Fléchon J. (Sous presse).

Wasserman F., MC Donald Th. F., ig63. Electron microscopic study of adipose tissue (fat organs) with special reference to the transport of liquids between blood and fat cells. Z. Zellforsch., 59, 326-357.

Weiss J. M., I955. The role of Golgi complex in fat absorption as studied with the electron microscope with observations on the cytology of duodenal absorptive cells. J.exper. Med., 102, 775-788.

Wotron R. M., I963. Lipid absorption. Int. Rev. Cylol., 15, 399-420.

ZETTERQViST H., I956. The ultrastructural organization of the columnar absorbing cell of the mouse jejunum. Thesis, Karolinska Institutet, Stockolm, $83 \mathrm{p}$.

Abréviations employées sur le schéma et les électromicrographies

ca : couche apicale.

D : desmosome.

DN : région subnucléaire.

ei : espace intercellulaire.

Eim : espace intermicrovilleux.

L : lipides étalés.

La : acheminement des lipides.

Lc : lipides accolés à la membrane plasmique.

Ld : emplacement des lipides éliminés.

Le : lipides situés dans l'espace intermicrovilleux.

Lei : lipides situés dans l'espace intercellulaire.

Lp : pénétration des lipides à travers la membrane plasmique libre.

Lr : lipides à l'intérieur du réticulum.

$\mathrm{Lvg}$ : lipides à l'intérieur des vésicules golgiennes.

II : mitochondries.

$\mathrm{mb}$ : membrane "basale ".

$\mathrm{mp}$ : membrane plasmique.

mv : microvillosités.

$N$ : noyau.

$\mathrm{PN}$ : région nucléaire.

$\mathrm{RE}$ : réticulum endoplasmique.

RER: réticulum endoplasmique rugueux.

Rmp: liaison entre le réticulum et la membrane plasmique.

Smv : sommet des microvillosités.

$\mathrm{SN}$ : région supranucléaire. 
PL. I

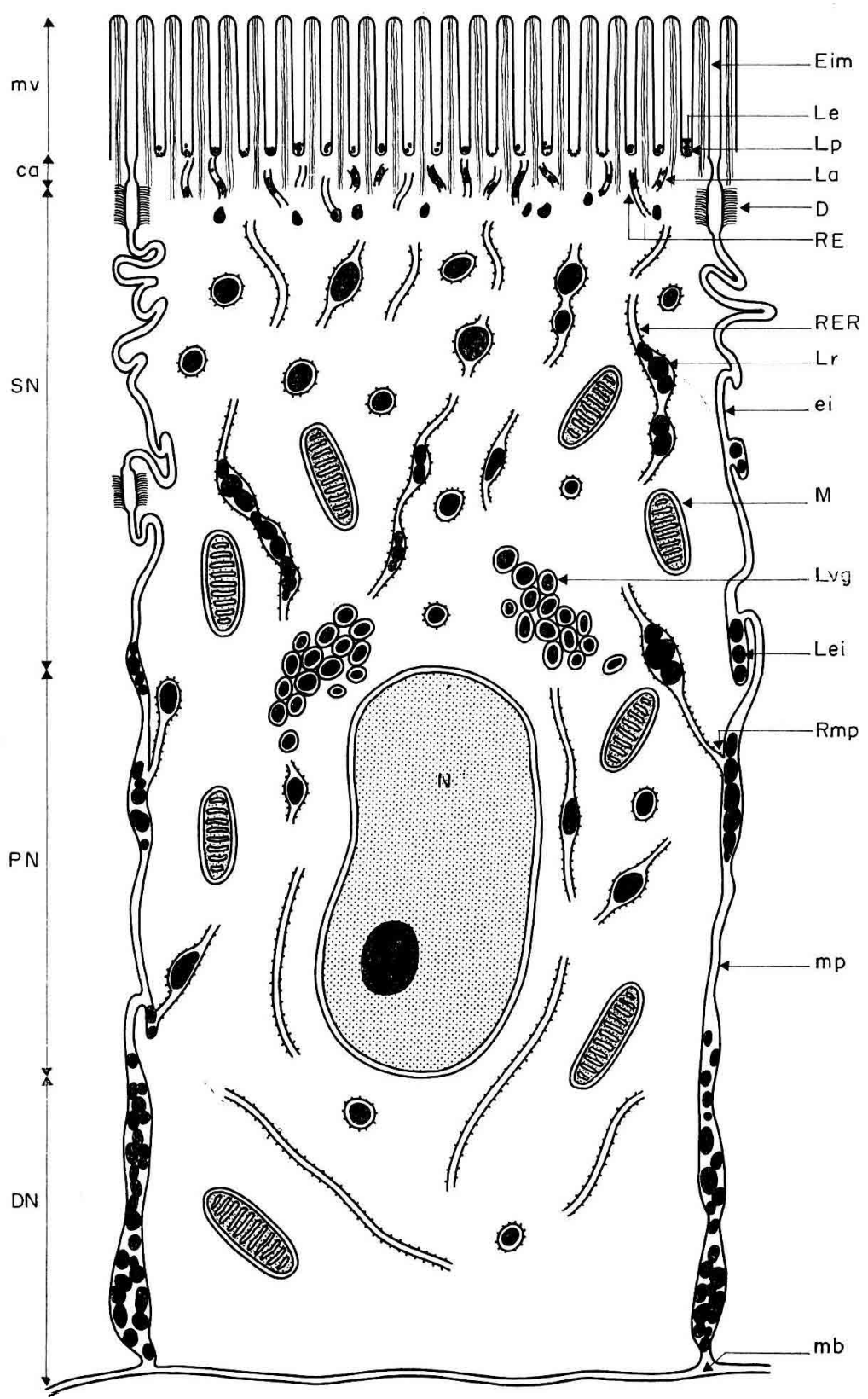

P'lanche I. Fig. I - Schéma de la cellule épithéliale absorbante de l'intestin grêle du Pore (pénétration et acheminement des lipides.) 


\section{PLANCHE, II}

FIG. 2

Sommet de villosité intestinale

Absorption des lipides. Les espaces intermicrovilleux sont remplis de lipides (Le). Formol-calcium; Noir Soudan B; $\times 4500$.

\section{FIG. 3}

Côtés latéraux de deux villosités contiguës

Absorption des lipides. Nombreuses particules de lipides dans le fond des espaces intermicrovilleux (Le). Formol-calcium; Noir Soudan B; $\times 4500$. 

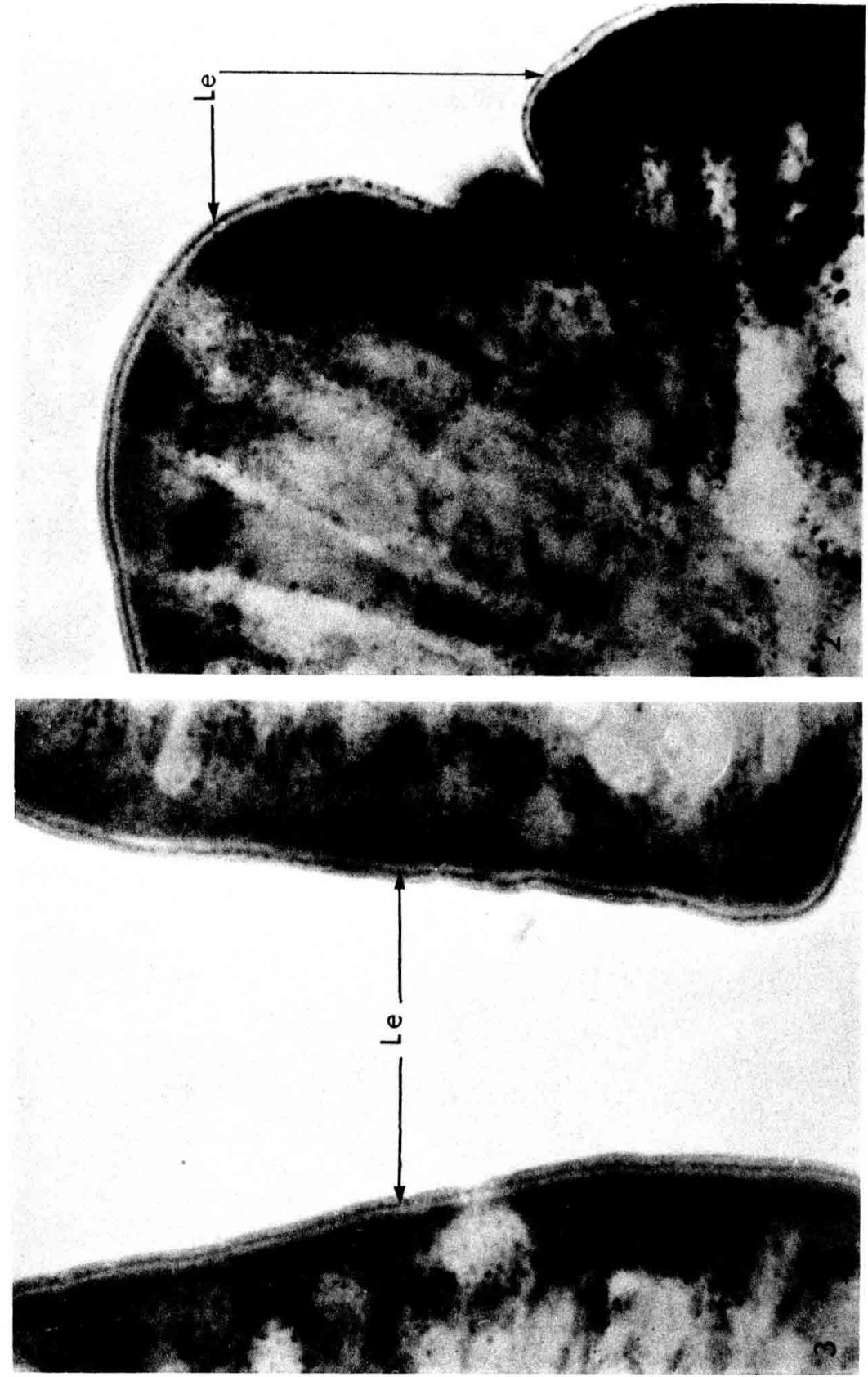


\section{PLANCHE III}

Fig. 4

Microvillosités et zone apicale de la cellule absorbante prélevée au sommet d'une villosité, coupe parallèle à la microvillosité. - Lipides au sommet des microvillosités (Smv), au moment de la pénétration au fond des espaces intermicrovilleux (Lp), pendant l'acheminement dans la zone apicale à l'intérieur du RE (La), puis à la limite de la zone apicale dans le RER (Lr). $\left(\mathrm{OsO}_{4}\right.$ à 2 p. 100 , méthacrylate prépolymérisé.

Acétate d'uranyle, citrate de plomb, $\times 30000)$.

FIG. 5

Microvillosités, zone apicale et partie de l'endoplasme de la cellule absorbante, coupe légèrement oblique sur le grand axe. - Lipides au sommet des microvillosités ( $\mathrm{Smv}$ ), accolés à la membrane des villosités (Le), au moment de la pénétration $(L p)$, en cours d'acheminement dans la zone apicale (La), puis à l'intérieur du RER dans l'endoplasme (Lr). Continuité entre le RE de la zone apicale et de l'endoplasme.

$\left(\mathrm{OsO}_{4}\right.$ à $2 \mathrm{p}$. 100 , méthacrylate prépolymérisé.

Acétate d'uranyle, citrate de plomb, $\times 30000$ ). 


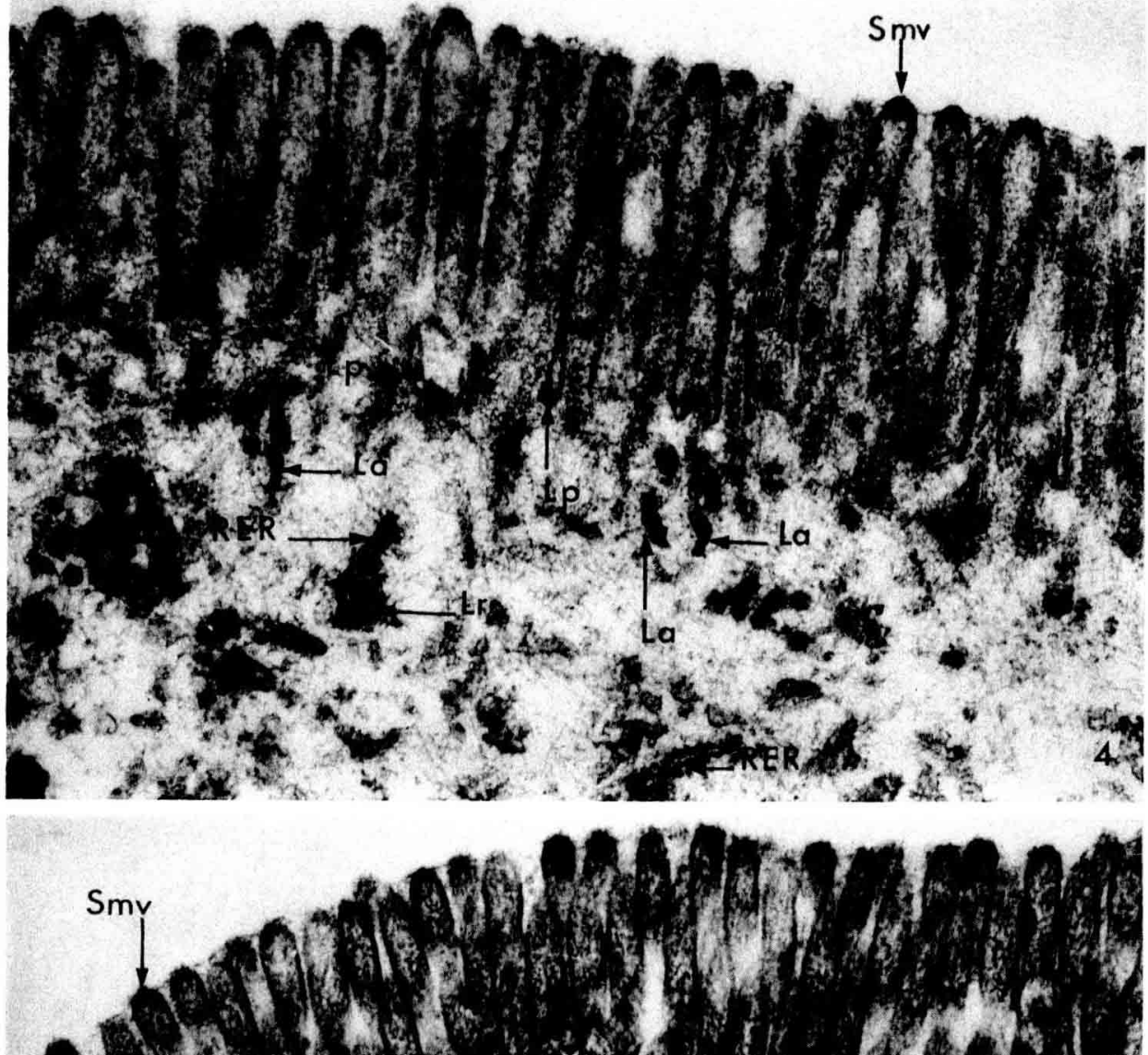

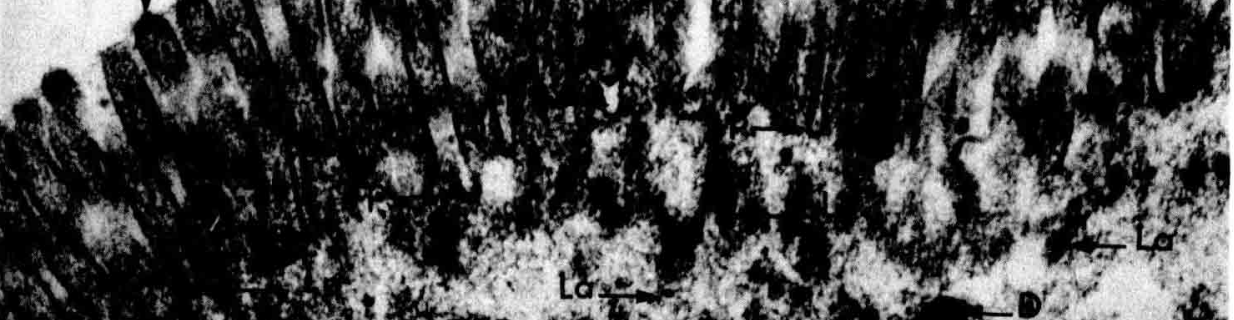

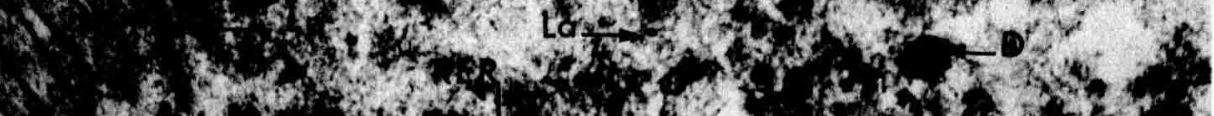

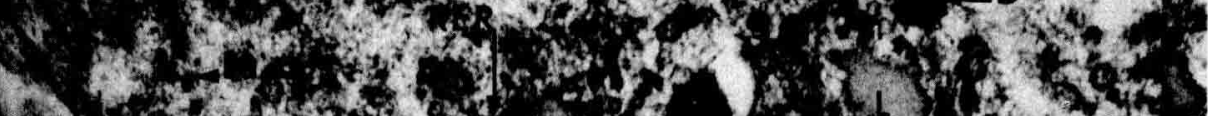

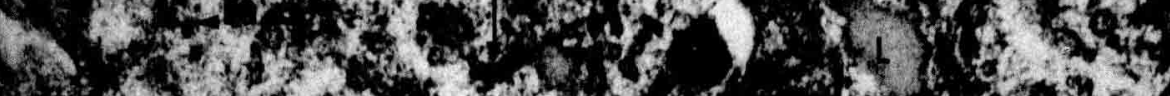

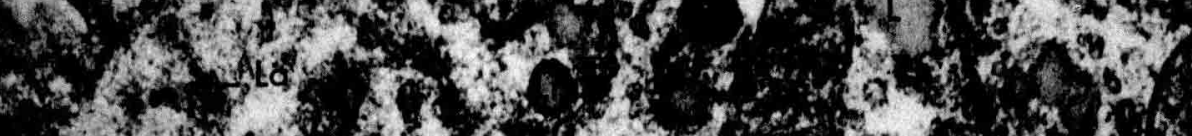

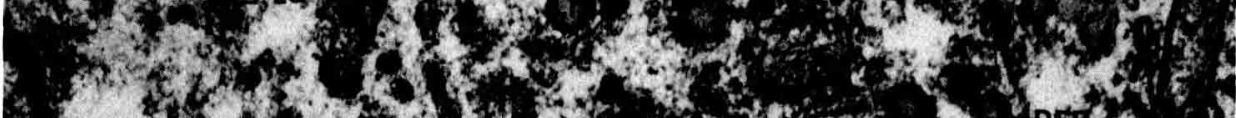

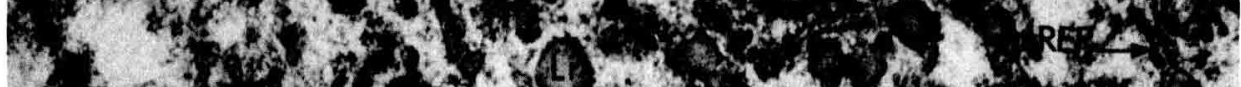

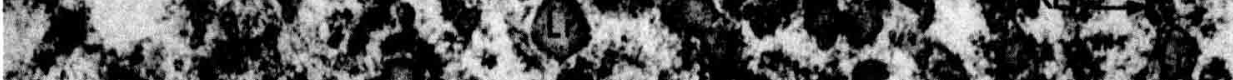

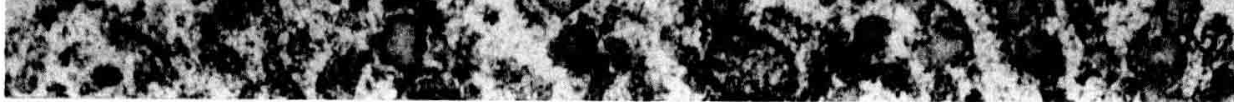




\section{PLANCHE IV}

\section{Fig. 6}

Microvillosités et zone apicale d'une cellule absorbante prélevée sur la partie médiane de la villosité au mème moment de l'absorption qu'aux figures 3 et 4 , coupe parallèle à l'axe de la microvillosité. - Lipides au sommet des microvillosités (Smv), accolés à la membrane plasmique (Lc), au fond des espaces intermicrovilleux (Le); emplacements d'où ils ont été éliminés (Ld), pénétration dans la membrane plasmique (Lp), acheminement dans la zone apicale (La), puis dans le RE de l'endoplasme où ils sont parfois étalés à l'intérieur du RER ou en gouttelettes (Lr).

$\left(\mathrm{OsO}_{4}\right.$ à 2 p. 100 , méthacrylate prépolymérisé.

Acćtate d'uranyle, citrate de plomb, $\times 30000$ ).

\section{Fig. 7}

Coupe oblique sur la microvillosité et zone apicale prélevée au même niveau et au même moment que sur la figure 6. - Montre les mêmes caractéristiques observées sous un angle différent. On distingue la substance osmiophile seulement sur les sommets des dernières rangées de microvillosités. Pour les autres sections, la triple couche de la membrane plasmique est distincte et on constate l'absence de substance osmiophile entre les deux lamelles opaques.

$\left(\mathrm{OsO}_{4}\right.$ à $2 \mathrm{p}$. 100, méthacrylate prépolymérisé.

Acétate d'uranyle, citrate de plomb, $\times 30000$ ). 

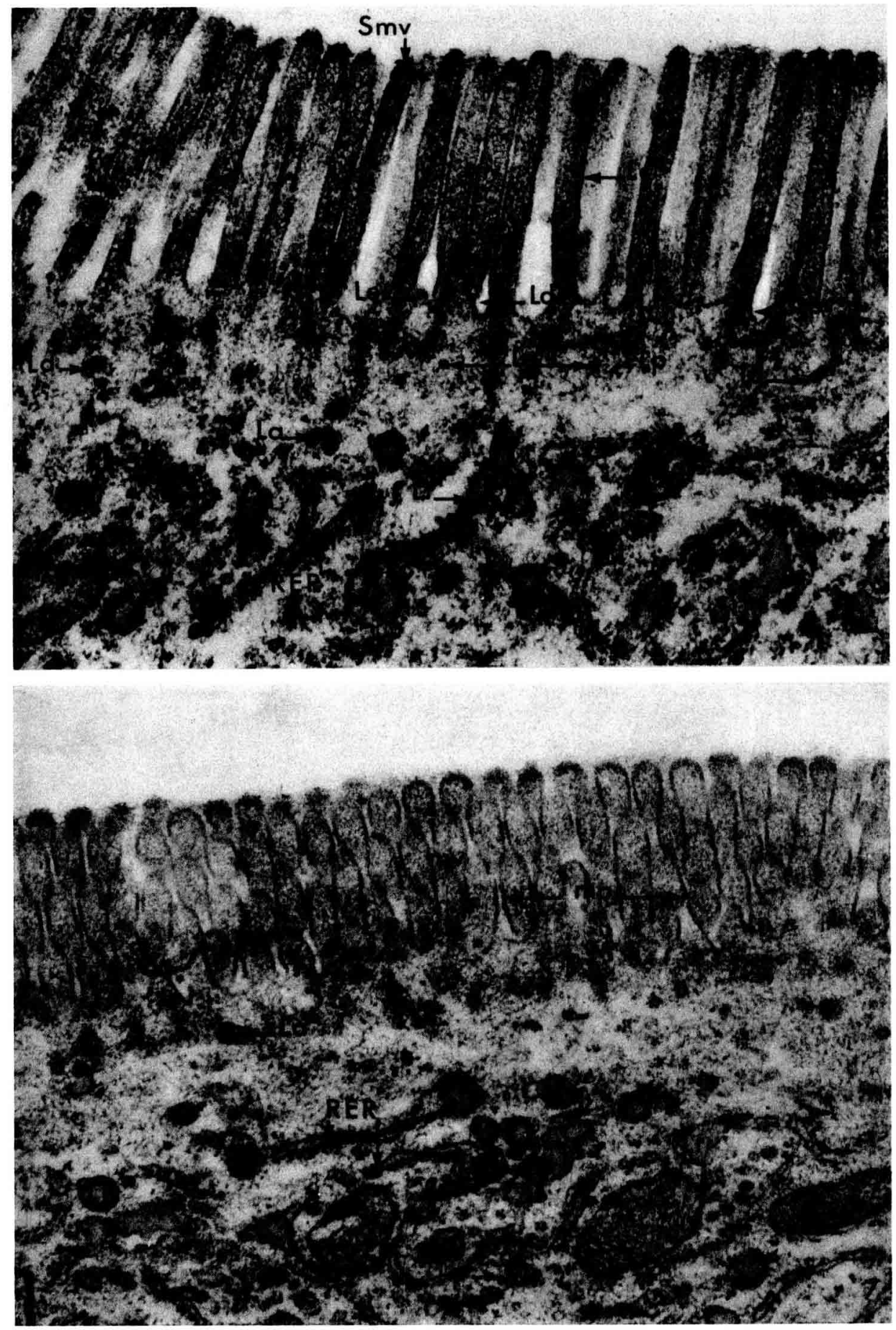


\title{
PLANC.HE V
}

\author{
FIG. 8
}

Coupe parallèle au grand axe de la cellule absorbante au niveau de la zone apicale et de l'endoplasme supranucléaire au moment de l'acheminement des lipides. - Pénétration des lipides ( $\mathrm{Lp}$ ), acheminement à travers la zone apicale (La) entourés d'une très fine membrane de RE, puis dans le RER (Lr).

$\left(\mathrm{OsO}_{4}\right.$ à 2 p. 100, méthacrylate prépolymérisé.

Acétate d'uranyle, citrate de plomb, $\times 30000$ ).

Fig. 9

Région de Golgi au moment du transit des lipides à travers les vésicules golgiennes. - Réticulum endoplasmique granuleux (RER), lipides à l'intérieur du RER (Lr), vésicules golgiennes remplies de lipides $(\mathrm{Lvg})$, noyaux $(\mathrm{N})$, membrane plasmique $(\mathrm{mp})$. $\left(\mathrm{OsO}_{4}\right.$ à 2 p. 100, méthacrylate prépolymérisé.

Acétate d'uranyle, citrate de plomb, $\times 30000$ ). 
n. Biol. anim., Bioch., Biophys., 1966, 6 (3). - M. VODOVAR, J. HLANZY PL. V

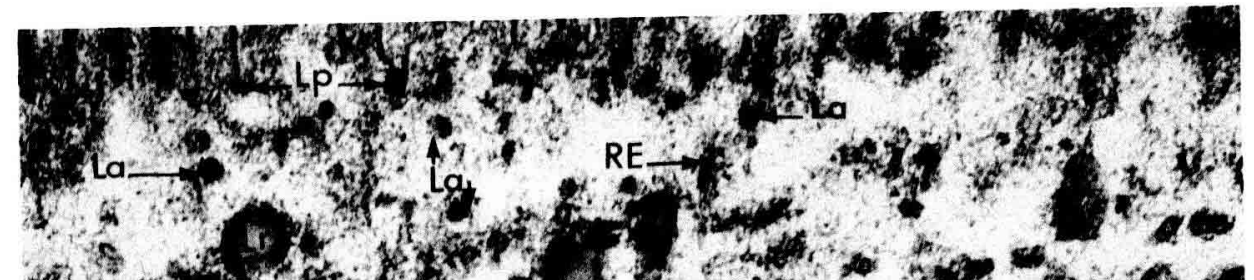
16)

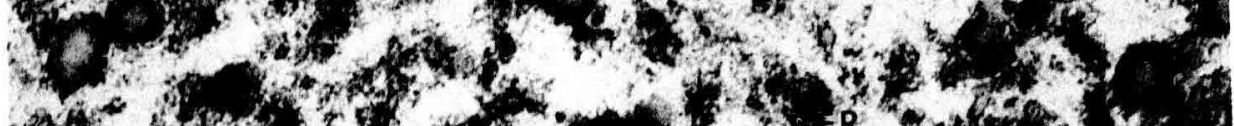

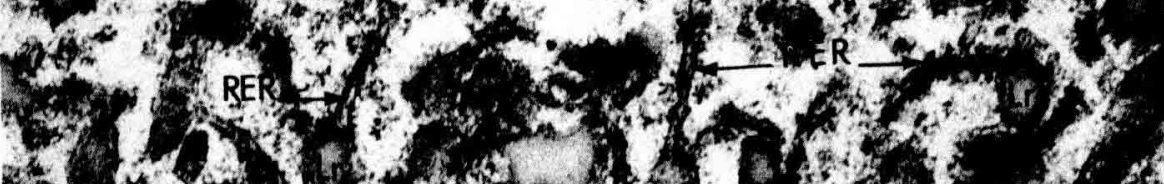
8.

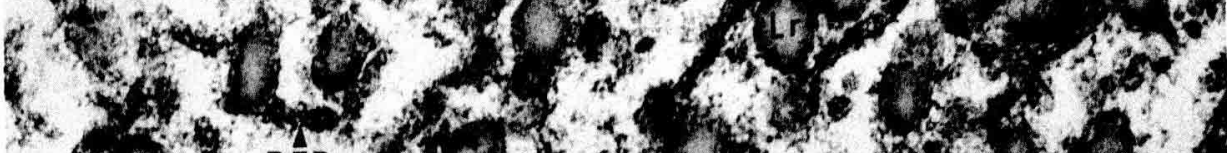

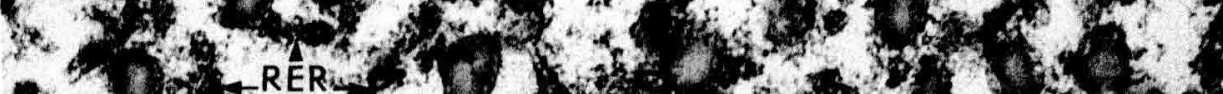

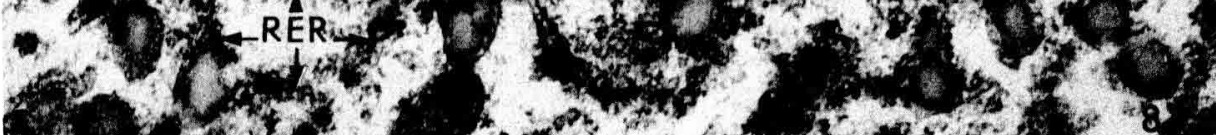

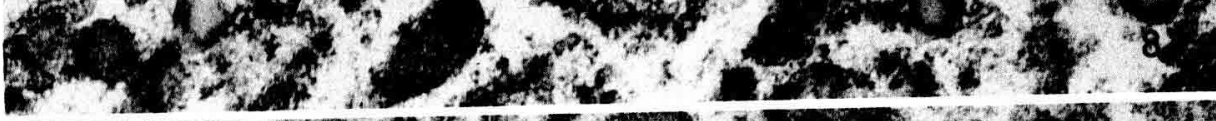

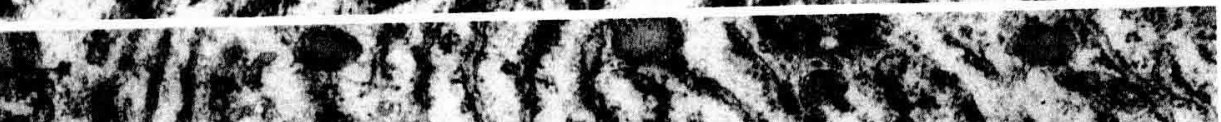

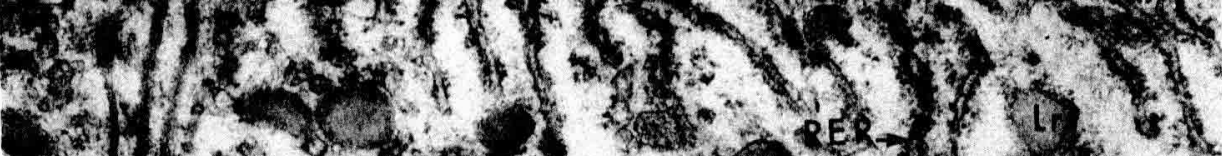

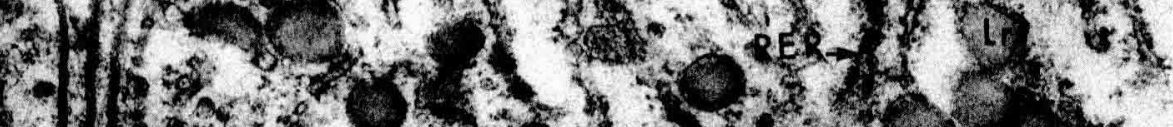
1.9

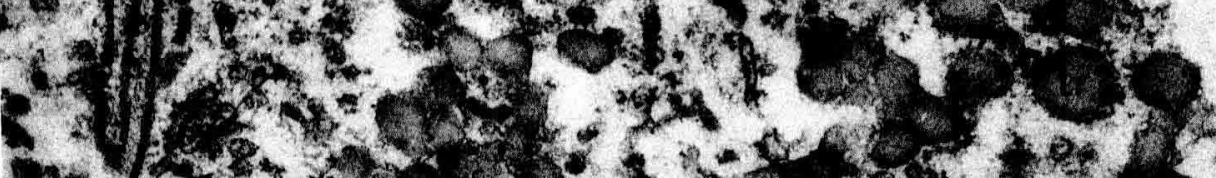

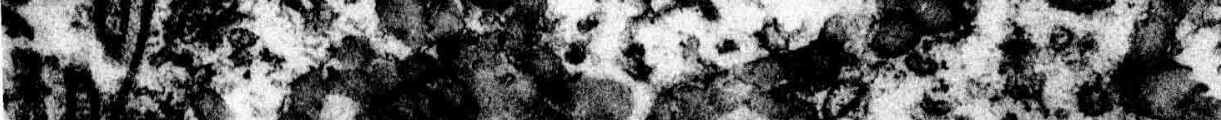

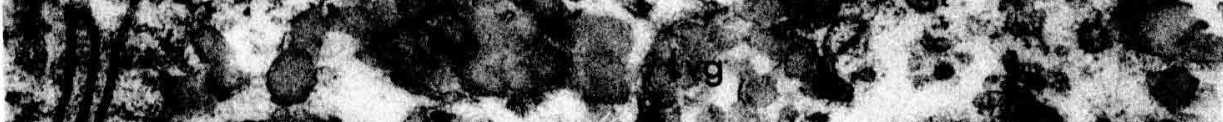

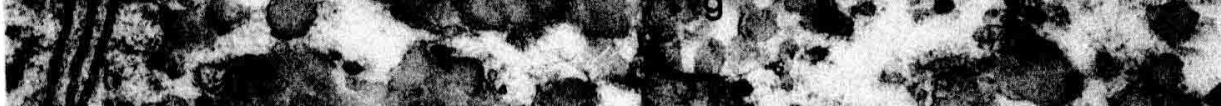

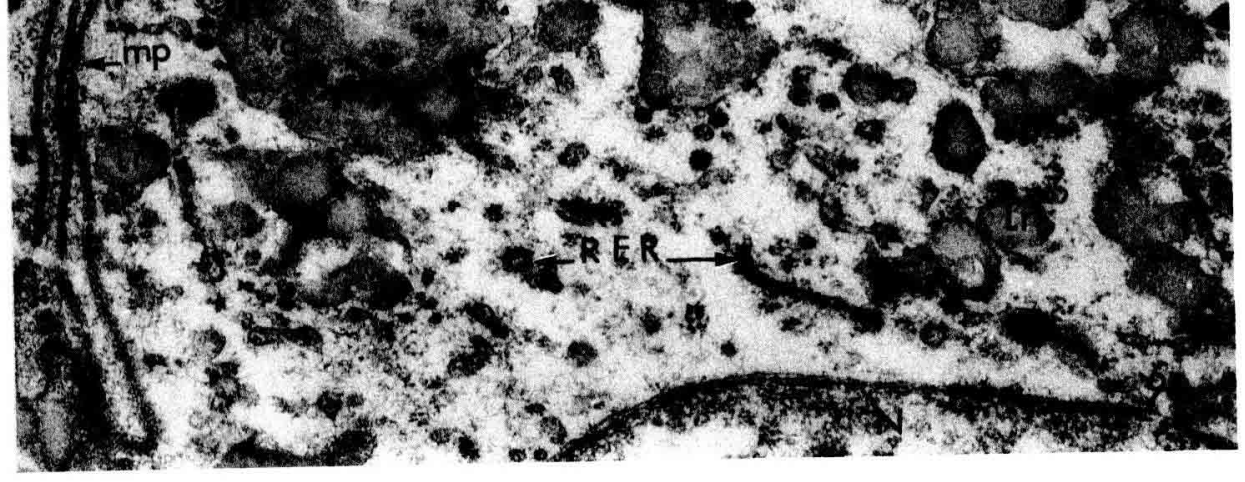




\title{
PLANCHE VI
}

\author{
FIG. 10
}

Coupe perpendiculaire au grand axe de la cellule absorbante au niveau du noyau. - La plupart des lipides se trouvent dans les espaces intercellulaires (Lei); de rares gouttelettes sont encore à l'intérieur du RER. Mithochondries (M), membrane plasmique (mp),

$\left(\mathrm{OsO}_{4}\right.$ à 2 p. 100, méthacrylate prépolymérisé.

Acétate d'uranyle, citrate de plomb, $\times 30000$ ).

\section{FIG. 11}

Coupe parallèle au grand axe de la cellule absorbante, région entre le noyau et la "basale ". - Lipides accumulés dans les espaces intercellulaires (Lei) près de la "basale " $(\mathrm{mb})$, noyau $(\mathrm{N})$, membrane plasmique latérale $(\mathrm{mp})$, mitochondries (M). $\left(\mathrm{OsO}_{4}\right.$ à 2 p. 100, méthacrylate prépolymérisé. Acétate d'uranyle, citrate de plomb, $\times 30000)$. 


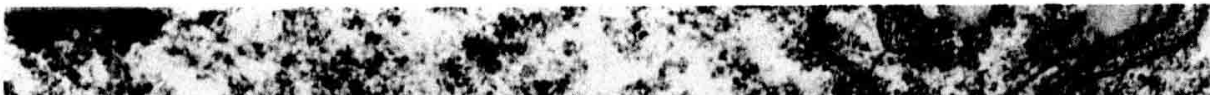

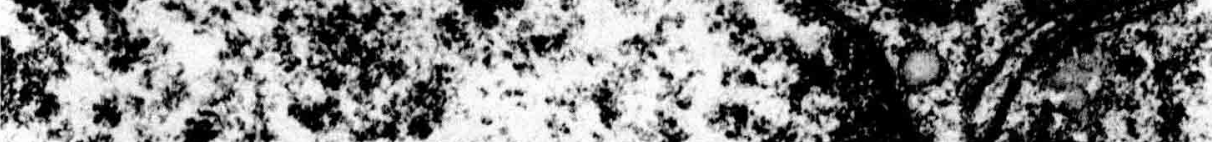

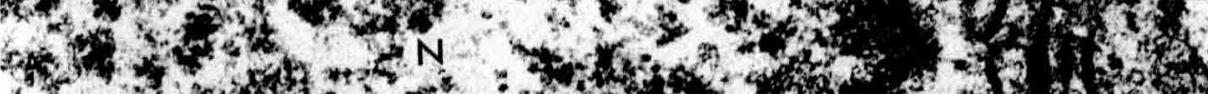

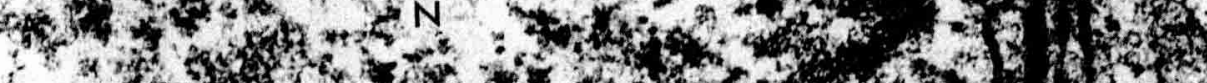

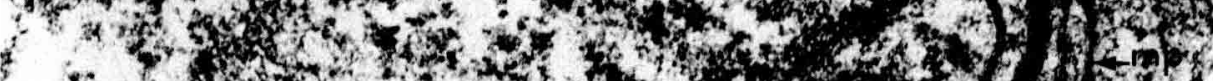

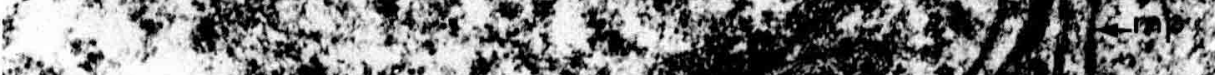

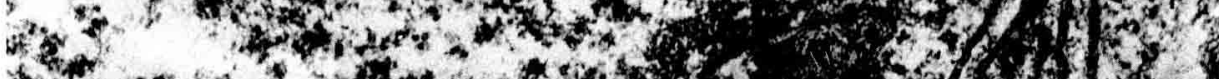

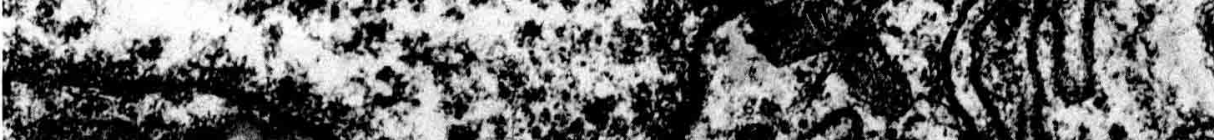

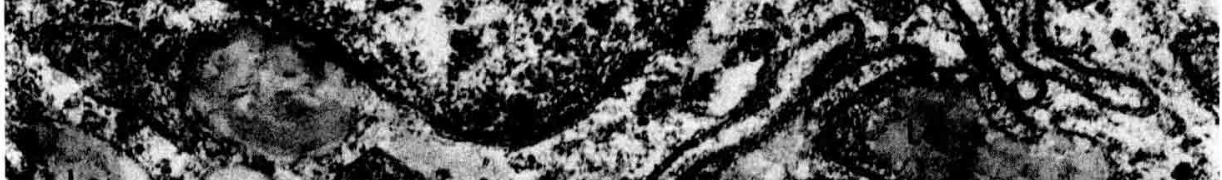

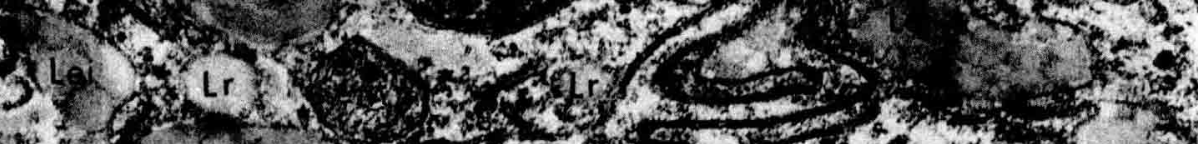

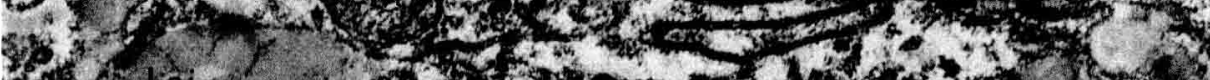

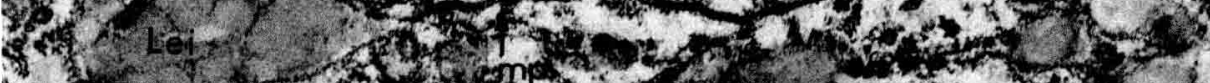
2. ${ }^{2}$.

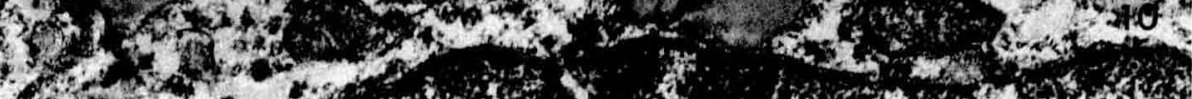

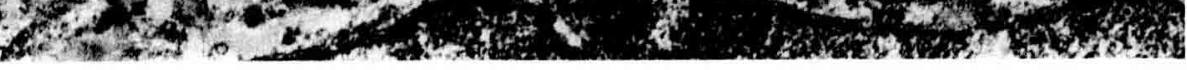

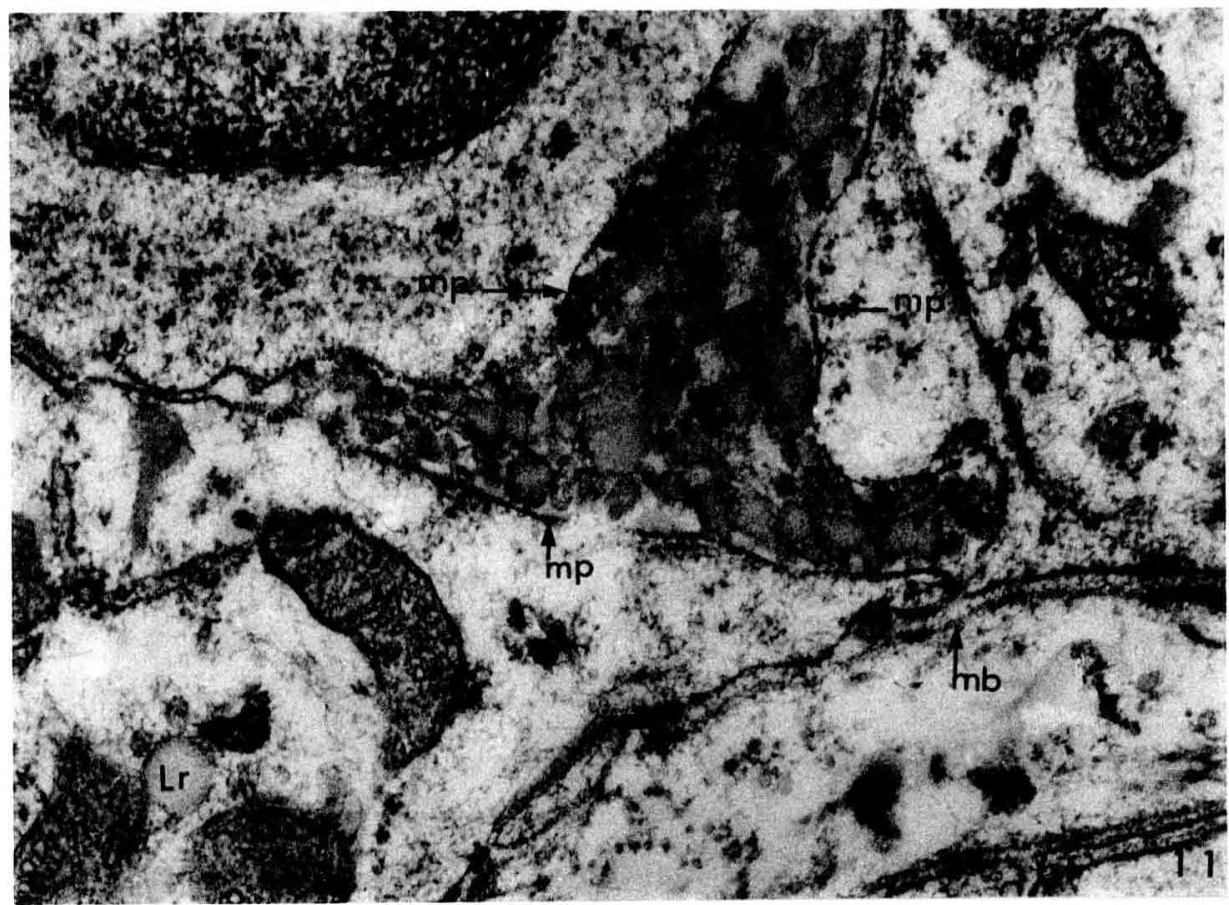

